\title{
Editor's Message to Special Issue on Network Services and Distributed Processing
}

\author{
TAMIO KIHARA ${ }^{1, a)}$
}

\begin{abstract}
"Network services" such as provided on the Internet have been integrated into many aspects of our lives and became indispensable infrastructure. However, the network services are always changing and evolving. Much kind of devices are getting connected to the network services, e.g., not only workstations and PCs, but also mobile phones, smartphones, home electronics, sensors, and so on. Moreover, network services now come to provide a space for communications like social network in addition to the traditional one-to-one or one-to-many communications such as e-mail or Web. Besides, new paradigms for the construction method of network services other than simple client-server are suggested like overlay-network, cloud computing, and so on. To keep the evolutions on the network services, cross-disciplinary study and forward-thinking study are required, in addition to the advancement of traditional network infrastructure, services, and applications. More specifically, the following innovative components including several new insights should be considered: e.g. smart network services based on the sensors and actuators, useroriented and context-aware content distribution services, collabo-
\end{abstract} ration environments based on large scale knowledge sharing, social deployment issues treating privacy and security, communication services under severe environment for wireless network such as rural areas, sparse device distribution areas, etc. The future of the network services can be actually achieved by harmonious integration between these new components and the existing network technologies, from physical layer of the network to application layer, treating much kind of technological and social issues.

The aim of this special issue is to investigate trends and promote the research area such as network platform technologies, service construction technologies, application technologies, new generation multimedia/distributed computing, and so on. Mainly the committee members from Special Interest Group on Distributed Processing System (IPSJ SIG-DPS) formed the editorial committee of this special issue as below. The Editorial Committee received 38 submitted papers and accepted 26 papers among many high-quality submissions (68\% acceptance rate). These papers were chosen based on the peer reviews and discussions in the Editorial Committee's meeting held in March, May, July and October 2012.

Papers from wide variety of research area that related to "Network Services and Distributed Processing" are submitted. The

Nippon Telegraph and Telephone Corporation, Yokosuka, Kanagawa 239-0847, Japan

a) kihara.tamio@lab.ntt.co.jp accepted papers include 4 papers related to "Online Entertainment", 4 papers related to "Network Security", 6 papers related to "Wireless Network and Mobile Network", 4 papers related to "Web Application Services", 3 papers related to "Cloud Computing" and 5 papers related to "Ubiquitous and Mobile Computing". Every accepted paper treats important research issue that is essential for the future network services. I hope that the special issue will further promote researches and making community between researchers in this area. Lastly, I would like to thank Yuuichi Teranishi, Editorial Board, and other Editorial Committee members for their enthusiastic contribution to the entire planning and reviewing processes.

\section{The Editorial Committee}

- Editor in-Chief: Tamio Kihara (NTT)

- Editorial Board: Yuuichi Teranishi (NICT)

- Editorial Committee: (Alphabetical Order) Shigeru Fujita (Chiba Institute of Technology)

Kentaro Go (Yamanashi Univ.)

Masataka Goto (Toshiba)

Koji Hashimoto (Iwate Prefectural Univ.)

Hiroshi Inamura (NTT DoCoMo)

Atsushi Kanai (Hosei Univ.)

Yuka Kato (AIIT)

Michiaki Katsumoto (Katsumoto Desing Laboratory)

Tomoya Kitani (Shizuoka Univ.)

Taku Konishi (NEC)

Takayuki Kushida (IBM)

Takuo Nakashima (Tokai Univ.)

Yoshinari Nomura (Okayama Univ.)

Masayuki Sakata (Hitachi)

Masaaki Noro (Fujitsu Lab.)

Yasuo Sambe (NTT Data)

Kazuto Sasai (Tohoku Univ.)

Fumiaki Sato (Toho Univ.)

Hiroshi Shigeno (Keio Univ.)

Tetsuya Shigeyasu (Hiroshima International Univ.)

Yoh Shiraishi (Future University Hakodate)

Yasuhisa Takizawa (Kansai Univ.) 
Journal of Information Processing Vol.21 No.2 186-187 (Apr. 2013)

Atsushi Tagami (KDDI R\&D Laboratories)

Yoshiaki Terashima (Mitsubishi Electric)

Minoru Uehara (Toyo Univ.)

Masashi Yamamuro (NTT Software)

Takuya Yoshihiro (Wakayama Univ.) 